\title{
क्षण
}

\section{Avaliação de um Ambiente Virtual de Aprendizagem de Imunologia}

\section{Adriano Theodoro da Silva*1, Gerlinde Agate Platais Brasil Teixeira², Claudia Marcia Borges Barreto ${ }^{3}$}

\footnotetext{
${ }^{1}$ Doutor/Professor/Pesquisador, Curso Técnico de Análises Clínicas/Escola Técnica Estadual de Saúde Herbert José de Souza/Fundação de Apoio à Escola Técnica do Estado do Rio de Janeiro. Rua Clarimundo de Melo, 847 - Quintino - RJ CEP 21311-280 - Brasil. adriano.theodoro1@gmail.com

2 Doutora/Professora/Pesquisadora, Departamento de Imunobiologia/Instituto de Biologia/Universidade Federal Fluminense Outeiro São João Batista - Campus Valonguinho - Centro, Niterói - RJ CEP 24210-130 Brasil.gerlinde.teixeira@gmail.com

3 Doutora/Professora/Pesquisadora, Departamento de Imunobiologia/Instituto de Biologia/Universidade Federal Fluminense Outeiro São João Batista - Campus Valonguinho - Centro, Niterói - RJ CEP 24210-130 Brasil. claudiamarcia@id.uff.br
}

\section{Resumo}

Descrevemos o processo de criação colaborativa de um ambiente virtual de aprendizagem (AVA) de acordo com os princípios das metodologias ativas de ensino e a sua avaliação. O ambiente virtual foi usado no apoio ao ensino presencial. Participaram dessa experiência híbrida de aprendizagem estudantes da disciplina Imunobiologia, oferecida no primeiro ano de um curso tradicional de graduação em Medicina. Ao término da disciplina, foi aplicado um questionário para avaliar a facilidade de uso e a percepção dos estudantes sobre a qualidade do aprendizado adquirido. A maioria dos estudantes que avaliaram a intervenção pedagógica afirmou que o ambiente foi fácil de usar, atendeu às expectativas de apoio ao ensino presencial e as atividades didáticas foram importantes para o aprendizado de habilidades como reflexão, pesquisa e discussão. Portanto, o ambiente virtual desenvolvido foi bem-sucedido e bem-aceito pelos estudantes.

Palavras-chave: Educação a distância, Moodle; Ensino de Imunologia, Mapa conceitual. 


\title{
Evaluation of a Virtual Learning Environment of Immunology
}

\begin{abstract}
We herein describe the process of collaborative creation and evaluation of a Virtual Learning Environment of Immunology according to the principles of active learning. The Web-based platform was used in support of face to face classroom teaching. First year Medicine undergraduate students attending "Immunobiology" course participated in this blended learning experience. At the end of the course, a questionnaire was applied to evaluate the ease of use and the students' perception of the quality of the acquired learning. Most students assessed the educational intervention said that the environment was easy to use, supports the classroom teaching and the educational activities were important for learning skills such as reflection, research and discussion. Therefore, the developed virtual environment was successful and well accepted by the students.
\end{abstract}

Keywords: Distance learning, Moodle, Immunology teaching, Concept map. 


\section{Introdução}

A gestão convencional dos currículos de cursos de graduação é geralmente pautada pelo bom senso ou pela falta dele, de acordo com a formação profissional do professor. Quando a base didático-pedagógica não é intencionalmente explicitada e plenamente executada pelo corpo docente, visando ao alcance do perfil profissional desejado, pode haver prejuízos na formação dos egressos. Assim, em geral, há a supervalorização do domínio cognitivo, não dando muita importância para o desenvolvimento de um conjunto de habilidades, tais como autonomia intelectual, tomada de decisões e comunicação, entre outras, capazes de promover a competência profissional desejada. Portanto, a formação profissional do estudante deveria ser pautada na plena implementação das orientações fornecidas pelas Diretrizes Curriculares Nacionais (DCN) dos cursos de graduação das áreas da saúde (Brasil, 2001; 2003; 2014), evitando o que temos chamado, a partir da nossa reflexão sobre o assunto, de "currículo da sorte". Nesse currículo, o estudante pode ou não ter a sorte de ser influenciado positivamente em sua formação de acordo com a qualidade do ensino a que foi submetido por um determinado grupo de docentes. Queremos dizer, com a expressão "currículo da sorte", que quando não há a gerência sobre o trabalho didático-pedagógico do professor, não é exercido um mesmo padrão de ensino para todos os estudantes. Segundo Debard (Debard, 2005), o ensino a distância oferece meio de padronizar o conteúdo produzido por especialistas e o compartilhamento de objetivos de aprendizagem por professores de uma mesma ou de várias instituições.

Nos últimos anos, o ensino mediado pelas tecnologias da informação e comunicação (TICs) tem sido utilizado para complementar currículos presenciais. Segundo Filatro (Filatro, 2008), é nesse contexto que se destacam os ambientes virtuais de aprendizagem (AVAs), onde se encontram reunidas ferramentas para comunicação (correio eletrônico e mensagens instantâneas); colaboração (fóruns e salas de batepapo) e compartilhamento de recursos (links que dão acesso a material didático e diversas mídias de comunicação).

No Brasil, a utilização dos AVAs para complementar o ensino presencial na área de saúde vem se expandindo desde a disseminação das TICs e da promulgação da Portaria no 4.059, de 10 de dezembro de 2004, do Ministério da Educação, que orienta as instituições de ensino superior a oferecer até vinte por cento de suas disciplinas na modalidade semipresencial (Brasil, 2004). 
Pesquisas recentes mostram que a apropriação das novas tecnologias pelos educadores nas escolas e universidades ainda é um desafio, pois, apesar de os indicadores de inclusão tecnológica aumentarem ano após ano, ainda não se sabe se os estudantes estão desenvolvendo uma aprendizagem ativa com o uso das tecnologias da informação e comunicação (Barbosa, 2014; Lima, 2011).

Embora exista uma grande quantidade de trabalhos sobre a implementação das TICs na formação em saúde (Cavalcante \& Vasconcellos, 2007), sua utilização inadequada apenas reforça o modelo de ensino tradicional, em que a predominância da ação do docente e a ênfase no conteúdo têm sido associadas à aprendizagem mecânica e à evasão escolar (Aguilar-da-Silva et al., 2009). Ao considerarmos um método de ensino que promova apenas a aprendizagem mecânica, isto é, quando as informações são aprendidas sem uma interação específica e relevante, ou de maneira arbitrária e literal (Ausubel, 1980), não promovendo sentido para os estudantes, é natural o desinteresse destes pelo estudo e, consequentemente, o abandono. Portanto, é crucial que um AVA seja desenvolvido sob uma abordagem de ensino que valorize a capacidade de trabalhar cooperativamente com base no pensamento crítico e científico, ao invés do simples exercício de memorização (Brasil, 2003). As metodologias ativas de ensino desenvolvem tanto o aspecto cognitivo quanto o atitudinal do estudante. Métodos como aprendizagem baseada em problemas, problematização e aprendizagem baseada em equipe (Mitre et al., 2008) têm alcançado sistematicamente resultados positivos (Turpen \& Finkelstein, 2010).

A posição do professor nos métodos ativos de ensino é de orientador, para que o estudante faça observações, reflexões, pesquisas e tome decisões de como solucionar problemas (Libardi, 2011). Tais habilidades intelectuais, por sua vez, são fundamentais para o exercício da autonomia intelectual, a tomada de decisões e a comunicação efetiva, entre outras capacidades apontadas nas DCN do curso de Medicina como necessárias à formação médica. Espera-se também que o graduando venha corresponsabilizar-se pela própria formação inicial, continuada e em serviço (Brasil, 2014).

Trabalhos recentes têm demonstrado que os AVAs que incorporam metodologias ativas de ensino têm efetivamente melhorado a compreensão de temas científicos pelos alunos. Estudos mostram que quando os estudantes interagem com um ambiente virtual 3D onde se explora a manipulação de variáveis para resolução de problemas há aumento 
da motivação e do aprendizado (Mayrose, 2012). Quando os ambientes virtuais utilizam algum fórum de discussão on-line e requerem interações colaborativas entre os estudantes, observa-se melhor compreensão de temas científicos de microbiologia (Ryan, 2013). Em outros estudos, a aprendizagem colaborativa permitiu aos estudantes o desenvolvimento de habilidades de pesquisa e aquisição de competência no acesso à informação (Johnson, 2013).

Durante os anos de 2011 e 2012, a Coordenadoria de Educação a Distância da PróReitoria de Graduação da Universidade Federal Fluminense (UFF) publicou dois editais para fomentar a criação de disciplinas a distância para cursos de graduação presenciais da instituição. O Departamento de Imunobiologia participou desses editais e, no primeiro, apresentou o projeto de desenvolvimento de AVA intitulado "Ensino de Imunologia a Distância", cujo desenvolvimento redundou em dois desafios: o primeiro foi envolver os docentes do Departamento de Imunobiologia na discussão sobre o que ensinar, com base na ementa existente, mas sob as orientações previstas nas Diretrizes Curriculares Nacionais para os cursos de graduação e nos respectivos perfis dos egressos (Brasil, 2001; 2003; 2014). O segundo desafio estava relacionado à adoção de uma nova concepção de ensino pelos professores na elaboração das aulas, isto é, proporcionar um ensino interativo no lugar da condição passiva do estudante, criando atividades na busca ativa pela solução de problemas.

No presente trabalho descrevemos o processo de criação colaborativa de um AVA de Imunologia desenvolvido sob alguns dos princípios das metodologias ativas de ensino - a cooperação, a pesquisa e a solução de problemas -, e a avaliação por estudantes do curso de graduação em Medicina da UFF.

\section{Metodologia}

Esta pesquisa consiste num estudo de caso com abordagem quantitativa e qualitativa, de natureza exploratória e interpretativa (Lüdke \& André, 1986). O AVA foi desenvolvido em quatro etapas, seguindo as orientações do Sistema de Desenho Instrucional: análise contextual; desenho instrucional; desenvolvimento (produção dos materiais didáticos digitais, seleção, implementação, registro e validação da plataforma educacional); e avaliação (Filatro, 2008). 


\subsection{Análise contextual}

$\mathrm{Na}$ análise contextual, realizada no início da disciplina, foi investigado o perfil dos estudantes quanto ao uso das tecnologias da informação e comunicação e aos seus hábitos de estudo, por meio de questionário semiestruturado confeccionado com perguntas fechadas com uso de escala (Likert, 1932) e quantificadas por frequência simples. O questionário foi elaborado com base no estudo sobre tecnologia educacional de Santa-Rosa e Struchiner (Santa-Rosa \& Struchiner, 2011) visando a, principalmente, identificar a finalidade e as características do ambiente virtual usado pelos estudantes.

\subsection{Desenho instrucional}

O desenho instrucional compreendeu o planejamento do ensino, que seguiu a ementa oficial da disciplina. Entretanto, seu objetivo geral, as estratégias e os recursos de aprendizagem foram orientados pelo perfil do egresso e o conjunto de conhecimentos, habilidades e atitudes presentes nas DCN do curso de Medicina (Brasil, 2014). O currículo do curso de Medicina da UFF prevê a inserção precoce do estudante nos diferentes cenários da prática médica, o que exige o uso de habilidades intelectuais como observação, reflexão, análise e crítica, entre outras, que nem sempre estão desenvolvidas nos recém-ingressados. Portanto, é importante que essas habilidades sejam promovidas por atividades didáticas interativas e significativas para os graduandos do primeiro ano.

O plano de ensino da disciplina Imunobiologia foi desenvolvido por uma equipe composta por oito docentes do Departamento de Imunobiologia do Instituto de Biologia da UFF que haviam participado de oficinas de desenvolvimento docente sobre as metodologias ativas de ensino. Assim, seria uma oportunidade de pôr em prática, de forma orientada, os princípios aprendidos. Com a intenção de promover o trabalho cooperativo para a construção do AVA intitulado Ensino de Imunologia a Distância, foi solicitado pela coordenadora do projeto que:

1. os professores construíssem um mapa conceitual - "ferramenta gráfica para organizar e representar o conhecimento" (Novak \& Cañas, 2008) - relativo ao ensino de Imunologia considerando o seguinte norteador: estudar a fisiologia do Sistema Imunológico na perspectiva da manutenção da 
homeostase do organismo em interação com o meio, da construção sóciohistórica do conhecimento e da aplicação profissional;

2. os professores identificassem os principais conceitos e eixos orientadores para o ensino de Imunologia.

Após a discussão em grupo dos mapas conceituais construídos individualmente, foi gerado um mapa conceitual final (Figura 1). O objetivo da implementação deste no desenho da estratégia didático-pedagógica implementada no AVA foi definir, em consenso, os principais conceitos e eixos orientadores que deveriam compor o ensino de Imunologia.

\subsection{Seleção do ambiente virtual de aprendizagem}

A seleção do ambiente virtual de aprendizagem foi feita considerando um sistema que priorizasse a aprendizagem colaborativa baseada na pedagogia socioconstrutivista (Vigotski, 1998) o armazenamento de informações, a consulta a essas informações, a comunicação entre os usuários, o rastreamento de dados e a geração de relatórios sobre o progresso dos participantes (Filatro, 2008).

\subsection{Produção dos materiais didáticos}

Os materiais didáticos foram produzidos levando em consideração os princípios das metodologias ativas de ensino. A fim de orientar a elaboração das aulas foi oferecido aos professores material de leitura envolvendo os seguintes assuntos: Diretrizes Curriculares Nacionais para os cursos de graduação da área de Saúde (Brasil, 2001; 2003; 2014); metodologias ativas de ensino - estudo de caso (Mitre et al., 2008); taxonomia dos objetivos educacionais de Bloom (Ferraz \& Belhot, 2010) e confeç̧ão de material instrucional para EaD (Filatro, 2008). Coube a cada professor preparar duas aulas sobre temas do seu interesse, de acordo com os pressupostos didáticos e pedagógicos estabelecidos pelo grupo. Cada aula era composta por objetivos de aprendizagem e atividades intencionalmente relacionadas ao seu alcance. A prática do pensamento científico pelos estudantes foi estimulada pela formulação e confrontação de hipóteses explicativas individualmente e em grupo nas aulas prático-demonstrativas 
presenciais e por meio de questionamentos por alunos e professora em fórum assíncrono no AVA.

\subsection{Implementação e registro do AVA}

A implementação envolveu a disponibilização e organização dos materiais didáticos, assim como a determinação dos horários de início e fim das atividades de aprendizagem na plataforma educacional. A definição de papéis e privilégios dos usuários e a disponibilização da plataforma educacional aos estudantes foi de responsabilidade da equipe da CEAD. A coordenadora do projeto, professora das aulas práticas da disciplina, e o doutorando colaborador participaram da mediação dos fóruns. O colaborador também foi responsável pela implementação e comunicação com os estudantes.

O registro do AVA foi realizado sob a licença Creative Commons (Commons Corporation, 2011).

\subsection{Avaliação do AVA}

Ao término da disciplina, foi aplicado um questionário semiestruturado com uma pergunta fechada e outra aberta para verificar sob dois aspectos a qualidade do AVA criado. O primeiro era relativo à facilidade de uso. O segundo, referente à percepção do que é aprendizado significativo de Imunologia para os estudantes. Dessa forma, pretendemos revelar a contribuição do AVA para a tomada de consciência dos estudantes sobre a influência do novo processo de ensino no aprendizado deles. Para tal foram usadas as duas perguntas a seguir:

1. Avalie o ambiente virtual de aprendizagem de Imunologia quanto à facilidade de uso segundo a escala:
a. Fácil
b. Muito fácil
C. Difícil
d. Muito difícil

2. A intenção inicial deste projeto consistiu em criar um espaço virtual para apoiar o aprendizado significativo em Imunologia. Em sua opinião, tal objetivo foi alcançado? Justifique. 
As respostas às perguntas fechadas foram quantificadas por frequência simples; as respostas às perguntas abertas foram avaliadas pela análise de conteúdo (Bardin, 2011) por meio da qual foram identificados nos textos as palavras-chave, os sentidos e as categorias de resposta.

\section{Resultados e discussão}

\subsection{Análise contextual}

A avaliação do perfil de uso da internet foi realizada com 46 estudantes, todos matriculados na disciplina Imunobiologia, oferecida no segundo período do curso de graduação em Medicina da UFF. Na análise do perfil dos estudantes quanto ao uso das TICs, verificamos que $100 \%$ dos entrevistados acessam a internet com uma frequência maior que três vezes por semana, e $88 \%$ possuem internet com banda larga em suas residências. Quanto ao uso de serviços de comunicação, redes sociais e fóruns de discussão, $100 \%$ dos entrevistados relataram utilizar tanto os serviços de comunicação quanto as redes sociais, enquanto apenas $46 \%$ informaram participar de algum fórum de discussão. Com relação à frequência de uso da internet para estudo de assuntos relacionados à Imunologia, 4\% informaram que frequentemente acessam e $96 \%$ dos estudantes afirmaram que nunca ou raramente a acessaram.

Estes dados revelam que, apesar de a maioria dos estudantes estar ambientada com o uso dos recursos da internet, tal uso não apresenta características pertinentes ao ensino em ambiente virtual formal. A partir desses dados, podemos inferir que tanto as instituições de ensino básico como as de ensino superior não têm adotado esses recursos em sala de aula para beneficiar a aprendizagem. Isso pode ser devido à ausência de competências didáticas e pedagógicas essenciais para exploração das potencialidades das novas ferramentas das TICs para sala de aula (Felizardo \& Costa, 2012). De acordo com Salvador et al. (Salvador, Rolando, Almeida, \& de Mello, 2015), sem um programa de apoio docente não seria possível uma mudança de cultura no gerenciamento do ambiente de aprendizagem de acordo com a orientação socioconstrutivista. 


\subsection{Desenho instrucional}

O mapa conceitual final formulado colaborativamente pelos docentes (Figura 1) contou com os seguintes eixos orientadores da disciplina e temas correlatos:

- Dinâmica celular (seleção, cooperação, especificidade, diversidade, memória e repertório);

- Organização morfofuncional do Sistema Imunológico (tecidos, células e moléculas e ontogênese);

- Contexto sócio-histórico (história da Imunologia, contexto social e prática profissional). 


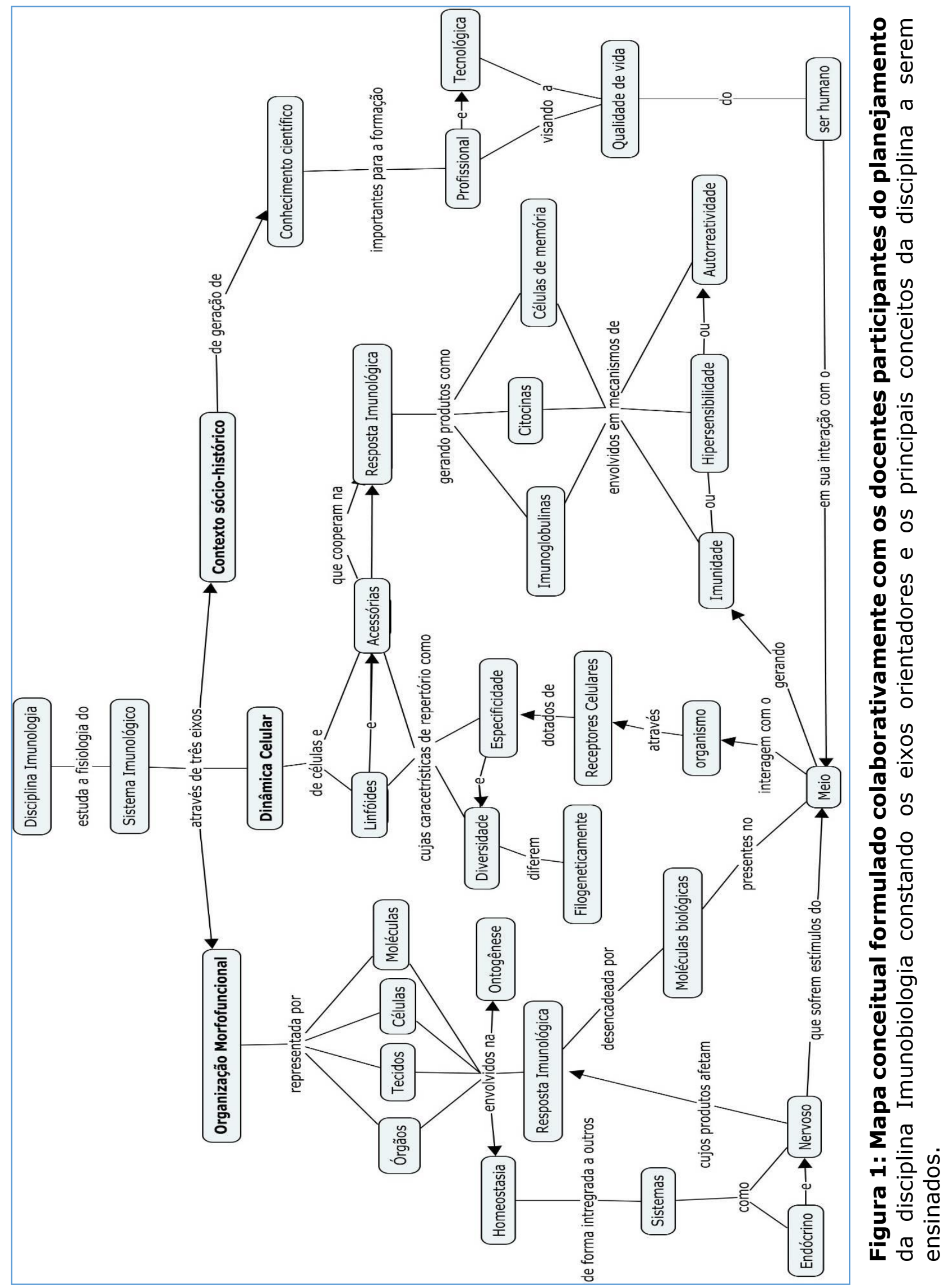




\subsection{Seleção do Ambiente Virtual}

O programa selecionado para criar o AVA foi o Modular Object Oriented Distance Learning (Moodle), um sistema de gerenciamento de aprendizagem que facilita a implementação de cursos a distância (Moodle, 2015). O programa é baseado na pedagogia socioconstrutivista e trata a aprendizagem como atividade social por meio de trabalho colaborativo em ambiente online (Figura 2). Criado em 2001 por Martin Dougiamas é distribuído gratuitamente sob licença General Public License (License, 2007).

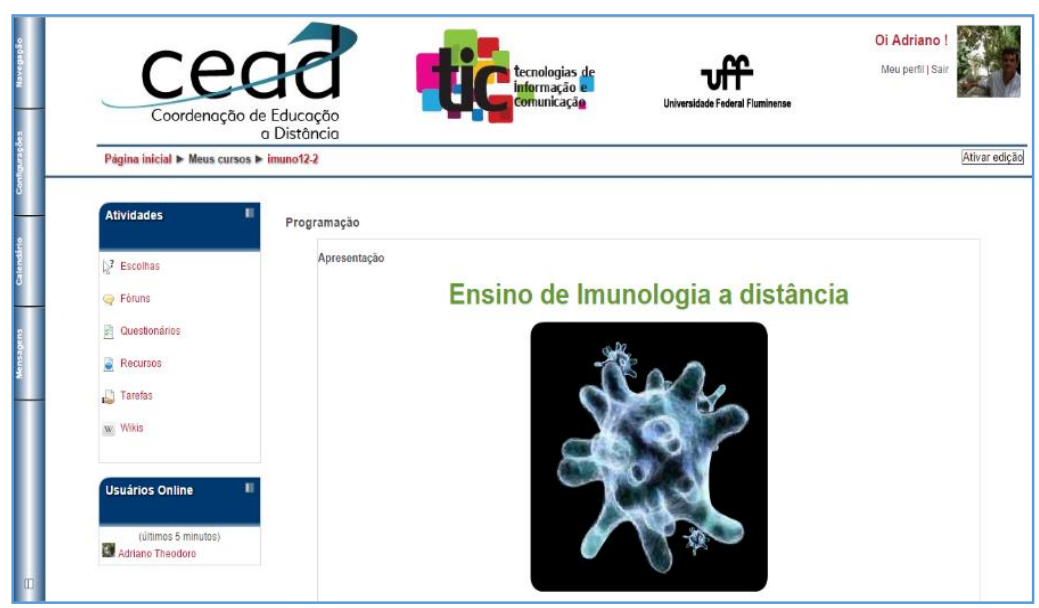

Figura 2. Página de Apresentação do AVA criado na plataforma Moodle.

\subsection{Produção dos materiais didáticos digitais}

A disciplina foi estruturada a partir de três unidades de ensino: (1) Estudo da Organização Morfofuncional do Sistema Imunológico; (2) Orquestração da Resposta Imunológica; e (3) Fisiologia do Sistema Imunológico e Integração com Outros Sistemas. $\mathrm{Na}$ Unidade 1, o objetivo foi estudar os componentes do Sistema Imunológico, a dinâmica celular e as características histológicas e anatômicas necessárias à compreensão das suas funções (Quadro 1). Na Unidade 2, o objetivo foi compreender os mecanismos celulares e moleculares envolvidos no desencadeamento da resposta imunológica e da imunidade (Quadro 2). Na Unidade 3, o objetivo foi relacionar o funcionamento do Sistema Imunológico com os sistemas endócrino e nervoso. Entretanto, as aulas só foram desenvolvidas, até o momento, para as Unidades de Ensino 1 e 2. 
Quadro 1 - Aulas relativas à Unidade de Ensino 1 - Estudo da Organização do Sistema Imunológico disponibilizadas aos estudantes durante o semestre letivo.

\begin{tabular}{|c|c|}
\hline Título das Aulas & Descrição das atividades de produção individual \\
\hline \multirow{3}{*}{$\begin{array}{l}\text { Medula } \\
\text { óssea }\end{array}$} & $\begin{array}{l}\text { Compare as características histológicas da medula óssea } \\
\text { de indivíduos jovens e adultos. }\end{array}$ \\
\hline & $\begin{array}{l}\text { Destaque a importância biológica das moléculas de } \\
\text { superfície que caracterizam os linfócitos } B \text {. }\end{array}$ \\
\hline & $\begin{array}{l}\text { Identifique os tecidos linfoides primários correspondentes } \\
\text { à medula óssea em diferentes grupos de animais, como } \\
\text { aves e mamíferos (coelhos, suínos e ruminantes). }\end{array}$ \\
\hline \multirow{3}{*}{ Timo } & $\begin{array}{l}\text { Relacione as características histológicas do estroma tímico } \\
\text { às principais etapas da diferenciação dos linfócitos T. }\end{array}$ \\
\hline & $\begin{array}{l}\text { Avalie a compreensão do significado de três palavras, } \\
\text { expressões ou siglas presentes no estudo acima. }\end{array}$ \\
\hline & $\begin{array}{l}\text { Identifique as consequências para a saúde dos indivíduos } \\
\text { na ausência do timo. }\end{array}$ \\
\hline \multirow{3}{*}{ Baço } & $\begin{array}{l}\text { Formule uma hipótese para o destino de restos celulares } \\
\text { de hemácias no sangue e de agentes infecciosos que } \\
\text { alcançam a circulação sanguínea e de metodologia para } \\
\text { testar a hipótese. }\end{array}$ \\
\hline & $\begin{array}{l}\text { Relate as semelhanças e as diferenças entre o método } \\
\text { pensado por você e o realizado por Biozzi. }\end{array}$ \\
\hline & $\begin{array}{l}\text { Identifique as principais consequências da esplenectomia } \\
\text { para a saúde do indivíduo. }\end{array}$ \\
\hline Anticorpo & $\begin{array}{l}\text { Você poderia formular uma hipótese explicativa para o fato } \\
\text { do Fab (produto do tratamento do anticorpo com a } \\
\text { papaína) se ligar ao antígeno, mas não ser capaz de } \\
\text { precipitá-lo? Compare as propriedades do Fab com a do } \\
\mathrm{F}\left(\mathrm{ab}^{\prime}\right) 2 \text { para fundamentar a sua resposta. }\end{array}$ \\
\hline \multirow[b]{2}{*}{$\begin{array}{l}\text { Sistema } \\
\text { complemento }\end{array}$} & $\begin{array}{l}\text { Faça uma pesquisa para identificar os fenômenos } \\
\text { biológicos desencadeados durante a ativação da cascata } \\
\text { enzimática do sistema complemento. }\end{array}$ \\
\hline & $\begin{array}{l}\text { Compare as diferentes vias de ativação do sistema } \\
\text { complemento visando identificar a sua importância } \\
\text { biológica. Identifique a componente chave comum às três } \\
\text { vias de ativação da cascata enzimática do sistema } \\
\text { complemento, que contribui para a geração de diferentes } \\
\text { fenômenos biológicos. }\end{array}$ \\
\hline
\end{tabular}

As aulas continham diferentes atividades orientadas por objetivos de aprendizagem explícitos na apresentação de cada uma delas; as atividades variaram de acordo com o nível cognitivo da Taxonomia de Bloom - conhecimento, compreensão, aplicação, análise, síntese e avaliação (Krathwohl, 2002). Dessa forma, há uma exigência explícita do trabalho intelectual a ser exercido pelos estudantes. A abordagem pedagógica e os recursos didáticos foram intencionalmente escolhidos visando à 
interatividade dos estudantes e ao desenvolvimento de habilidades intelectuais como pensamento crítico, reflexivo e científico. Foram criadas 10 aulas, totalizando 20 atividades, das quais 14 de produção individual (sendo nove obrigatórias) e duas de produção colaborativa. Os estudantes foram instruídos a realizar um total de quatro aulas no AVA como complementares à aula presencial; as demais ficaram disponíveis para uso livre.

Quadro 2 - Aulas relativas à Unidade de Ensino 2 - Orquestração da Resposta Imunológica disponibilizadas aos estudantes durante o período letivo.

\begin{tabular}{|c|c|}
\hline Título & Descrição das atividades de produção individual \\
\hline $\begin{array}{c}\text { Mecanismo } \\
\text { de imunidade } \\
\text { celular e } \\
\text { humoral }\end{array}$ & $\begin{array}{l}\text { Com base no texto, relacione os produtos da resposta } \\
\text { humoral e celular que podem ser efetivos no controle da } \\
\text { infecção, considerando as características de colonização } \\
\text { dos agentes biológicos no hospedeiro, isto é: extracelular, } \\
\text { obrigatoriamente intracelular ou de hábito facultativo. } \\
\text { O tétano é causado por uma neurotoxina (tetanoespasmina) } \\
\text { produzida pelo Clostridium tetani, bactéria extracelular, } \\
\text { anaeróbica e de pouca invasividade. A soroterapia é a } \\
\text { principal medida adotada clinicamente para evitar a } \\
\text { evolução da doença. Qual é a explicação científica para esse } \\
\text { fato? }\end{array}$ \\
\hline Título & Descrição das atividades assíncronas \\
\hline \multirow[b]{2}{*}{$\begin{array}{l}\text { Padrão de } \\
\text { resposta } \\
\text { imunológica }\end{array}$} & $\begin{array}{l}\text { Relacione a natureza dos componentes dos agentes } \\
\text { biológicos às células e às citocinas envolvidas com o } \\
\text { desenvolvimento dos padrões de resposta imunológica } \\
\text { mediados por linfócitos Th1, Th2 e Th17 estão adequados e } \\
\text { completos. }\end{array}$ \\
\hline & $\begin{array}{l}\text { Suponha que você tenha que investigar o caso de } \\
\text { imunodeficiência em uma criança que apresenta infecções } \\
\text { pulmonares recorrentes por pneumococos. Você já havia } \\
\text { solicitado exame para HIV (ELISA e PCR) e o resultado foi } \\
\text { negativo, estando em níveis normais os percentuais de } \\
\text { células T CD4 e CD8 constatados por citometria de fluxo. } \\
\text { Considerando as informações acima e os mecanismos de } \\
\text { ativação de linfócitos, qual investigação você faria para } \\
\text { identificar a causa da imunodeficiência da criança? }\end{array}$ \\
\hline
\end{tabular}




\subsection{Implementação e registro do ambiente virtual}

A plataforma digital foi carregada no servidor da coordenação de Educação a Distância - CEAD, http://www.cead.uff.br/, que é um órgão vinculado à Pró-Reitoria de Graduação - PROGRAD/UFF, e registrada sob a licença de Atribuição, Uso Não Comercial e Não a Obras Derivadas da Creative Commons (Commons Corporation, 2011).

\subsection{Avaliação do ambiente virtual de aprendizagem}

Participaram da experiência híbrida de ensino e, portanto, da avaliação do AVA 46 estudantes de uma turma da disciplina Imunobiologia do curso de Medicina da UFF durante o primeiro semestre de 2013. Ao todo foram verificados 10.690 registros de acesso, com $57 \%$ dos estudantes realizando 10 a 200 acessos, $30 \%$ de 200 a 400 acessos e $13 \%$ de 400 a 800 acessos. Verificamos uma frequência alta de estudantes participantes das atividades das aulas, recomendadas como complementares ao ensino presencial, relativas ao aprendizado da função dos órgãos linfoides como medula óssea $(75 \%)$, timo $(97 \%)$ e baço $(81 \%)$. As outras atividades, não especificamente recomendadas, como as relacionadas ao estudo dos anticorpos e sistema complemento foram desenvolvidas por $17 \%$ e $16 \%$, respectivamente. Ao verificarmos a frequência de estudantes que participaram das atividades relacionadas ao estudo do padrão de resposta imunológica e mecanismo celular e humoral de imunidade, contabilizamos $40 \%$ e $55 \%$, respectivamente.

$\mathrm{Na}$ avaliação da facilidade de uso do AVA, constatamos que $25 \%$ dos estudantes consideraram o ambiente muito fácil, $67 \%$ consideraram o ambiente fácil, $8 \%$ difícil; nenhum estudante considerou o ambiente muito difícil de ser usado. Considerando que $92 \%$ dos estudantes nunca haviam usado tecnologia digital no apoio ao ensino presencial de Imunologia, nós avaliamos que o AVA foi adequadamente construído. A facilidade de uso do AVA é fundamental, pois a dificuldade em identificar e compreender as tarefas pode gerar desinteresse e consequentemente a evasão dos estudantes. Segundo De Oliveira et al. (De Oliveira et al., 2005) a facilidade de uso é crucial no emprego ou não de programas educativos, principalmente por exigirem do estudante atenção aos elementos de interface a serem manipulados para realizar as atividades de aprendizagem. 
A análise das respostas de 19 estudantes para a pergunta aberta - "A intenção inicial deste projeto consistiu em criar um espaço virtual para apoiar o aprendizado significativo em Imunologia. Em sua opinião, tal objetivo foi alcançado?" - gerou o seguinte resultado: $73 \%$ concordaram, $21 \%$ concordaram parcialmente e apenas um estudante não concordou (6\%). A análise qualitativa das justificativas dos estudantes concordantes revelou o efeito positivo sobre o aprendizado por meio de declarações como "bastante eficiente na compreensão da matéria" e "proposta pedagógica eficiente frente aos métodos tradicionais de ensino". Evidenciamos nas justificativas apenas uma categoria relativa à percepção dos estudantes quanto a uma nova abordagem didáticopedagógica, que coincidiu com a proposta do AVA criado colaborativamente. As atividades intencionalmente planejadas para promover a interatividade, a reflexão e a pesquisa na solução de problemas foram identificadas nos seguintes exemplos de fragmentos de justificativas dos estudantes: "forma mais interativa de ensino da Imunologia", "podemos aprender com discussões", "uma alternativa para reforçar conteúdo, preparar para reflexão e manter contato com a matéria", "é muito eficaz na construção autônoma do nosso conhecimento" e "os fóruns ajudam a debater e discutir os conceitos e a aplicação na clínica médica".

\section{Conclusão e considerações finais}

Concluímos que o processo de construção do AVA Ensino de Imunologia a Distância foi bem-sucedido, sendo criadas 10 aulas contemplando atividades diversificadas e pautadas por princípios das metodologias ativas de ensino reconhecidos pelos estudantes. O AVA foi considerado de fácil acesso e atendeu às expectativas dos estudantes quanto ao apoio ao ensino presencial, promovendo a satisfação deles com o aprendizado adquirido, atingindo, portanto, o objetivo proposto no seu desenvolvimento.

Neste momento estamos concluindo a pesquisa que objetiva avaliar a influência do AVA criado sobre o modo de estudar e de aprender dos estudantes.

\section{Agradecimento}

Este trabalho foi financiado pelo Plano de Desenvolvimento Institucional da Universidade Federal Fluminense. 


\section{Referências bibliográficas}

Aguilar-da-Silva, R. H., Perim, G., Abdala, I., Costa, N., Lampert, J., \& Stella, R. (2009). Abordagens pedagógicas e tendências de mudanças nas escolas médicas. Rev Bras Educ Med, 33(Supl 1), 53-62.

Ausubel. (1980). Psicologia educacional: Rio de Janeiro: Interamericana. Tradução para português, de Eva Nick et al., da segunda edição

de Educational psychology: a cognitive view.

Barbosa, A. (2014). Pesquisa sobre o uso das tecnologias de informação e comunicação nas escolas brasileiras: tic educação 2013. São Paulo: Comitê Gestor da Internet no Brasil. Disponível em: http://www.cetic.br/media/docs/.

Bardin, L. (2011). Analise de conteudo. 6-ed. Lisboa (Por): Edicoes, 70.

Brasil, M. (2001). Parecer CNE/CES 1.133/2001, de 7 de agosto de 2001: Diretrizes curriculares nacionais dos cursos de graduação em enfermagem, medicina e nutrição. Disponível em: http://portal.mec.gov.br/dmdocuments/ces1133.pdf.

Brasil, M. (2003). Diretrizes Curriculares Nacionais dos Cursos de Graduação em Biomedicina. Disponível em: http://portal.mec.gov.br/cne/.

Brasil, M. (2004). Portaria no 4059 de 10 de dezembro de 2004. Trata da oferta de disciplinas na modalidade semi-presencial em cursos superiores já reconhecidos. Diário Oficial da União de 13 de dezembro de 2004., Seção 1, p.34. Disponível em: http://portal.mec.gov.br/sesu/.

Brasil, M. (2014). Parecer CNE/CES 116/2014, de 6 de junho de 2014: Diretrizes curriculares nacionais do curso de graduação em medicina. Disponível em: http://portal.mec.gov.br/.

Cavalcante, M. T. L., \& Vasconcellos, M. M. (2007). Tecnologia de informação para a educação na saúde: duas revisões e uma proposta. Ciência \& Saúde Coletiva, 12(3), 611-622.

Commons Corporation, C. (2011). The power of open. Acesso em 14 de outubro de 2015. Disponível em: http://thepowerofopen.org/.

De Oliveira, J. M. P., Fernandes, C. T., Ramos, E. S., Jacinto, A., Vilela, F., \& Ajukas, D. (2005). Processo de Prototipação da Interação em STI que Utiliza a Aprendizagem Baseada em Problemas como Proposta Pedagógica. Paper presented at the Anais do Simpósio Brasileiro de Informática na Educação. Disponível em: http://www.br-ie.org/pub/index.php/sbie/article/view/423.

Debard. (2005). Science and society - The influence of the Internet on immunology education. Nature Reviews Immunology, 5(9), 736-740. Disponível em: http://www.nature.com/nri/journal/v5/n9/full/nri1687.html.

Felizardo, M. H. V., \& Costa, F. A. (2012). A Formação de Professores e a Integração Das Tic No Currículo: Com Que Formadores? . Paper presented at the Actas do II 
Congresso TIC e Educação. Disponível em: http://ticeduca.ie.ul.pt/atas/pdf/55.pdf.

Ferraz, A., \& Belhot, R. V. (2010). Taxonomia de Bloom: revisão teórica e apresentação das adequações do instrumento para definição de objetivos instrucionais. Gest. Prod., São Carlos, 17(2), 421-431.

Filatro, A. (2008). Design instrucional na prática: São Paulo: Pearson Education do Brasil. Johnson, K. (2013). Facilitating Cooperative Learning In Online and Blended Courses: An Example from an Integrated Marketing Communications Course. American Journal of Business Education (Online), 6(1), 33. doi: http://dx.doi.org/10.19030/ajbe.v6i1.7481.

Krathwohl, D. R. (2002). A revision of Bloom's taxonomy: An overview. Theory into practice, 41(4), 212-218. Disponível em: http://www.unco.edu/cetl/sir/stating outcome/documents/Krathwohl.pdf.

Libardi, D. A. (2011). O papel do professor universitario na construção do conhecimento. Revista de Educação, 13(15), 9-26. Disponível em: http://pgsskroton.com.br/seer/.

License, G. P. (2007). Free Software Foundation. Acesso em 14 de outubro de 2015, Disponível em: http://fsf.org/.

Likert, R. (1932). A technique for the measurement of attitudes. Archives of psychology. Lima, A. (2011). TIC na educação no Brasil: O acesso vem avançando. E a aprendizagem. Bilíngue Pesquisa sobre o uso das tecnologias de informação e comunicação no Brasil: TIC Educação, 27-33. Disponível em: http://cetic.br/media/docs/publicacoes/2/tic-educacao-2011.pdf.

Lüdke, M., \& André, M. E. (1986). Pesquisa em educação: abordagens qualitativas: Editora Pedagógica e Universitária.

Mayrose, J. (2012). Active Learning Through The Use Of Virtual Environments. American Journal of Engineering Education (AJEE), 3(1), 13-18. doi: http://dx.doi.org/10.19030/ajee.v3i1.6885.

Mitre, S. M., Siqueira-Batista, R., Girardi-de-Mendonça, J. M., Morais-Pinto, N. d., Meirelles, C. d. A. B., Pinto-Porto, C., ... Hoffmann, L. M. A. (2008). Metodologias ativas de ensino-aprendizagem na formação profissional em saúde: debates atuais. Ciênc saúde coletiva, 13(2), 2133-2144. doi: http://dx.doi.org/10.19030/ajee.v3i1.6885.

Moodle. (2015). Moodle Documentation. Acesso em: 01 de maio de 2015, disponível em: http://moodle.org/.

Novak, J. D., \& Cañas, A. J. (2008). The theory underlying concept maps and how to construct and use them. Florida Institute for Human and Machine Cognition, 2008.

Ryan, R. S. (2013). The effect of online discussion forums on student learning and student perception of learning in a science course at the community college level. Disponível em: http://aquila.usm.edu/cgi/. 
Salvador, D. F., Rolando, L. G. R., Almeida, C. d. M., \& de Mello, J. A. (2015). Mudança de cultura no uso de tecnologias educacionais: estudo de caso no modelo semipresencial do Cederj. EAD em FOCO, 5(1). doi:10.18264/eadf.v5i1.313.

Santa-Rosa, J. G., \& Struchiner, M. (2011). Tecnologia educacional no contexto do ensino de histologia: pesquisa e desenvolvimento de um ambiente virtual de ensino e aprendizagem. Rev Bras Educ Med, 35(2), 289-298. doi:http://dx.doi.org/10.1590/S0100-55022011000200020.

Turpen, C., \& Finkelstein, N. D. (2010). The construction of different classroom norms during Peer Instruction: Students perceive differences. Physical Review Special Topics-Physics Education Research, 6(2), 0201231-02012322.doi:

http://dx.doi.org/10.1103/PhysRevSTPER.6.020123.

Vigotski, L. S. (1998). A formação social da mente: São Paulo: Martins Fontes. 


\section{Levantamento do perfil de ensino e aprendizagem dos estudantes}

Pergunta 1: Você possui internet em sua residência?
a. $\operatorname{Sim}$
b. Não

Pergunta 2: Qual seu tipo de acesso à internet?
a. Discado
b. Banda Larga

Pergunta 3: Com qual frequência você utiliza a internet?

a. 1 a 3 vezes por semana b. Acima de 3 vezes por semana c. Nunca

Pergunta 4: Qual serviço de comunicação você mais utiliza?

a. Skype b. MSN c. Twitter d. Outros e. Não utilizo nenhum serviço de comunicação

Pergunta 5: Qual rede social você mais utiliza? social
a. Orkut
b. Facebook
c. Outros
d. Não utilizo nenhuma rede

Pergunta 6: Qual fórum ou grupo de discussão você mais utiliza?

a. Yahoogroups b. Googlegroups c. Não participo de nenhum fórum ou grupo de discussão d. Outros

Pergunta 7: Como você está habituado a estudar para as provas? Outros
a. Na semana das provas
b. Nos fins de semana
c. Todos os dias
d. grupo?

Pergunta 8: Como você está habituado a estudar, individualmente ou em
a. Individualmente
b. Em grupo
c. Outros

Pergunta 9: Você costumava, no Ensino Médio, usar a internet para estudar assuntos relacionados à Imunologia?
a. Sempre
b. Frequentemente
c. Raramente
d. Nunca 